\title{
CIRCE: A Dedicated Storage Ring for Coherent THz Synchrotron Radiation
}

\author{
J.M. Byrd, Michael C. Martin, W.R. McKinney, D.V. Munson, H. Nishimura, D.S. \\ Robin, F. Sannibale, R.D. Schlueter, W.G. Thur, J.Y. Jung, and W. Wan \\ Advanced Light Source Division, Lawrence Berkeley National Laboratory, 1 Cyclotron \\ Road, Berkeley, CA 94720, USA
}

\begin{abstract}
We present the concepts for an electron storage ring dedicated to and optimized for the production of stable coherent synchrotron radiation (CSR) over the far-infrared terahertz wavelength range from $200 \mu \mathrm{m}$ to about one $\mathrm{cm}$. CIRCE (Coherent InfraRed CEnter) will be a $66 \mathrm{~m}$ circumference ring located on top of the ALS booster synchrotron shielding tunnel and using the existing ALS injector. This location provides enough floor space for both the CIRCE ring, its required shielding, and numerous beamlines. We briefly outline a model for CSR emission in which a static bunch distortion induced by the synchrotron radiation field is used to significantly extend the stable CSR emission towards higher frequencies. This model has been verified with experimental CSR results. We present the calculated CIRCE photon flux where a gain of 6 - 9 orders of magnitude is shown compared to existing far-IR sources. Additionally, the particular design of the dipole vacuum chamber has been optimized to allow an excellent transmission of these far-infrared wavelengths. We believe that the CIRCE source can be constructed for a modest cost.
\end{abstract}

\section{Keywords}

Synchrotron, Coherent, Terahertz, Far-IR, Source, Flux, Design 


\section{Introduction}

The terahertz $(\mathrm{THz})$ and sub- $\mathrm{THz}$ region of the electromagnetic spectrum lies between the infrared and the microwave [1]. This boundary region is beyond the normal reach of optical and electronic measurement techniques and sources associated with these betterknown neighbors. Our recent research has demonstrated a much higher power source of $\mathrm{THz}$ radiation: coherent synchrotron radiation (CSR) [2]. Besides offering high power, CSR enables broadband optical techniques to be extended to nearly the microwave region [3], and it inherently has sub-picosecond pulses. As a result, new opportunities for scientific research and applications are enabled across a diverse array of disciplines: condensed matter physics, medicine, manufacturing, and space and defense industries. CSR will have a strong impact on $\mathrm{THz}$ imaging, spectroscopy, femtosecond dynamics, and driving novel non-linear processes.

CSR is emitted by bunches of accelerated charged particles when the bunch length is shorter than the wavelength being emitted. When this criterion is met, all the particles emit in phase, and a single-cycle electromagnetic pulse results with an intensity proportional to the square of the number of particles in the bunch [4, 5]. It is this quadratic dependence that can produce colossal intensities even with fairly low beam currents [2]. Until recently CSR has not typically been observed in synchrotron storage rings because the electron bunch lengths are longer than the vacuum chamber height, so full-bunch coherent emission is at wavelengths beyond the waveguide cut-off and is therefore suppressed. The first observations of CSR from storage rings were of quasichaotic bursts of intensity caused by density modulations in unstable electron bunches [69]. While studies of this 'bursting' phenomenon have provided glimpses into the powers available with CSR, the unstable nature of the emission makes this a problematic $\mathrm{THz}$ source for scientific measurements. We have experimentally verified a model [10] predicting where this unstable bursting regime will occur [9] and can use this experience to design future sources to avoid bursting instabilities entirely.

Recently, stable CSR has been produced at the BESSY-II storage ring $[11,12]$ and the first scientific measurements using this CSR source were recently reported [3]. This 
stable CSR emission is not driven by any instabilities, yet it extends to higher frequencies than predicted by a simple full-bunch coherence model [12]. We outline the model we have developed to explain this phenomenon [13]. The combination of the experimentally verified models for stable CSR as well as the threshold of which current levels will produce bursting instabilities allows us to fully design and optimize a new CSR source that will produce copious amounts of stable far-IR, THz and sub- THz, synchrotron radiation.

\section{Production of Stable CSR}

As mentioned in the introduction, CSR has been observed from storage rings having two different forms of emission versus time: bursting and stable. At the ALS we have made extensive measurements of far-IR bursts caused by micro-modulations of the electron bunch distribution. We showed that the beam current threshold for the onset of these bursts agrees very well with a model of self-amplified spontaneous bunch dynamics driven by the CSR itself [9]. The experimental data and theoretical model results agree with no adjustable parameters. Additionally this model fits very well with bursting instability thresholds carefully measured at BESSY[14]. Therefore the model is quite robust to different synchrotrons and we can now use it to set an upper limit to the amount of current per bunch that can be stored in a future ring optimized for stable CSR.

Stable CSR from a storage ring was recently observed at BESSY [11, 12]. Streak camera measurements at BESSY indicated a bunch length of $\sim 1 \mathrm{~mm}$ while the coherent emission was observed to extend to $\sim 200 \mu \mathrm{m}$ wavelength. This observation is in disagreement with the CSR emission predicted for the normal Gaussian bunch shape, and implies a distortion in the electron bunch distribution. We have developed a detailed model for how CSR can self-induce a static deformation of the electron bunch profile thus generating CSR to higher frequencies than the nominal bunch length would allow [13]. Figure 1 shows how in this model the synchrotron wakefield generated by the CSR itself acts on the electron bunch such that the bunch gets a sharper leading edge which coherently emits to frequencies three times higher than the undistorted bunch. This model qualitatively predicts the observed spectra published by BESSY [11, 12] and 
allows us to carefully plan how a dedicated ring can be designed to maximize the CSR output while remaining below the bursting threshold.

\section{CIRCE Ring Design \& Beamline Extraction}

We now want to design CIRCE, a ring-based source exploiting this effect. In the design of such a source one of the fundamental requirements is the stability of the CSR emission. We have therefore optimized the various machine parameters to maximize the CSR emission while keeping the beam current below the bursting threshold $[9,13]$. We maximize the CSR bandwidth by designing a vacuum chamber with a very low frequency cutoff to allow for transmission of wavelengths out to $\sim 1 \mathrm{~cm}$, and by tuning the ring parameters to increase the static distortion of the electron bunch profile as discussed above to extend the CSR emission to shorter wavelengths. A double-bend achromat lattice was chosen to allow significant flexibility in getting to low momentum compaction, a key requirement to produce $\sim 1$ picosecond bunch lengths. Lowering the energy of the electron beam to a moderate value of $600 \mathrm{MeV}$, helps shorten the bunches but is not so low that it becomes difficult to maintain the stability of the electron beam. A higher-frequency RF system (1.5 GHz superconducting) also aids in shortening the electron bunches. Additional families of magnets were added to allow fine control of higher order components of the momentum compaction.

The optimized size of a ring having all these parameters is almost the same size at the existing Advanced Light Source (ALS) booster ring, which allows an opportune use of available space at the ALS. We propose to build the CIRCE ring on top of the existing booster shielding and make use of the booster for full energy injection, as shown in a 3 dimensional computer aided design (CAD) drawing in Figure 2. The booster shielding tunnel is a single, poured in place, 1 meter thick concrete structure. As such it is quite stable with accelerometer measurements showing that the top surface is as stable as the main ALS experimental floor. We plan to build a compact shielding block system very close to the CIRCE ring to allow for the beamlines to fit on the same booster ring shielding surface. This proximity of the beamlines to the source points in the bending magnets gives another advantage for keeping the vibration pickup on the photon beam to 
a minimum. Initial studies of the ALS infrastructure have found no showstoppers for building the CIRCE ring on this location.

Once the CSR is emitted by the electron bunches, it is important to efficiently collect this powerful $\mathrm{THz}$ light. We accomplish this by choosing short radius bending magnets which allow the placement of the first collecting mirror close to the source point, and by a uniquely designed vacuum chamber shown in the upper panel of Figure 3. The opening of this vacuum chamber follows the photon beam profile allowing us to collect the light from a full $60 \%$ of the electron beam's trajectory within the bending magnet. The first mirror located at the large end of this chamber will collect $300 \mathrm{mrad}$ horizontally by 140 mrad vertically. This very large vertical collection angle captures $95 \%$ of the emitted synchrotron radiation at a $1 \mathrm{~mm}$ wavelength, and essentially $100 \%$ collection of shorter wavelengths. The lower panel of Figure 3 schematically shows how the first mirror will deflect the beam 90 degrees downward, and then will be followed by beamline optics to re-image the light into a spectrometer or other endstation equipment located directly outside the shield walls.

The unusual design of the vacuum chamber means that we must concern ourselves with trapped electromagnetic field modes, usually referred as high order modes (HOM), that could influence the stability of the electron beam. Accurate simulations of these fields in the chamber have shown only small couplings between these modes and the electron beam. Additionally, the large photon beam aperture after the first mirror allows the HOM to propagate outside the main chamber where they can be easily damped by RF absorbers. Bench measurements on a full-scale mock chamber (shown in the photograph in Figure 3) have completely confirmed the simulations results so that we are confident that HOM will not be an issue for the beam stability[15].

\section{CIRCE Performance}

Using the machine parameters for the CIRCE ring in our model for the CSR production from distorted electron bunches, we calculated the $\mathrm{THz}$ flux produced by the CIRCE ring. The results are plotted along with conventional synchrotron and thermal far-IR 
sources in Figure 4. The three different CIRCE curves represent three (of many) possible machine configurations. To maximize the CSR bandwidth toward the short wavelengths, we need to decrease the electron bunch length while keeping the distribution distortion to the maximum allowed by the model stability criterion for the bursting instability [13]. At shorter bunch lengths, the threshold for bursting becomes lower so the total number of electrons and therefore the total CSR power emitted is limited (red curve in Figure 4). To increase the total power emitted we can lengthen the bunch, while maintaining the maximum distortion. This means the CSR doesn't extend to as short of wavelengths, but it allows for increasing the number of electrons in the bunch (green curve). Since the intensity emitted goes like the number of electrons squared, the flux can be significantly increased in this method.

Comparing to existing synchrotron and thermal far-IR sources, Figure 4 shows that CIRCE will have between a 6 and 9 order of magnitude increase in average flux. A list of parameters for the CSR light generated by CIRCE is given in Table 1. The emitted light will come in transform limited pulses with durations of $\sim 300$ femtoseconds at the shorter wavelengths, and at a repetition rate tunable between $4.5 \mathrm{MHz}$ and $1.5 \mathrm{GHz}$ (varied simply by choosing which electron bunches are filled). Since these pulses are single cycle, they should be coherent with each other, and therefore we are investigating a pulse-stacking resonator. This would allow particular beamlines to amplify the peak power of the pulses at the expense of repetition rate, and allow that user to select the timing of their pulses independent of the other beamlines and users at CIRCE.

\section{Conclusion}

CIRCE will be a revolutionary source for a traditionally difficult spectral region at the border between optics and electronics, namely the "THz-gap." We have explored the virtues of a small ring dedicated to the production of coherent far-infrared and $\mathrm{THz}$ radiation and have determined conclusively that such a machine will create a true leap forward for the field and is entirely feasible. The total estimated cost for building the ring, including the first several beamlines, is \$20 million. Just as conventional 
synchrotron radiation has been a boon to x-ray science, coherent synchrotron radiation may lead to many new innovations and discoveries in terahertz science.

\section{Acknowledgements}

We gratefully acknowledge the work of numerous colleagues at LBNL with many details of the CIRCE design its integration into the ALS facility. We also acknowledge fruitful collaborations with P. Kuske, G. Wüstefeld, and the rest of the CSR team at BESSY. This work was supported by the Laboratory Directed Research and Development Program of Lawrence Berkeley National Laboratory under the Department of Energy Contract No. DE-AC03-76SF00098.

\section{References}

1. Ferguson, B., Zhang, X. C., "Materials for terahertz science and technology", Nature Materials, 1 (2002), 26.

2. Carr, G.L., M.C. Martin, W.R. McKinney, K. Jordan, G.R. Neil, and G.P. Williams, "High-power terahertz radiation from relativistic electrons", Nature, 420 (2002), 153.

3. Singley, E.J., M. Abo-Bakr, D.N. Basov, J. Feikes, P. Guptasarma, K. Holldack, H.W. Hübers, P. Kuske, M.C. Martin, W.B. Peatman, U. Schade, and G. Wüstefeld, "New scientific opportunities with intense coherent $\mathrm{THz}$ synchrotron radiation: Measuring the Josephson plasma resonance in $\mathrm{Bi} 2 \mathrm{Sr} 2 \mathrm{CaCu} 2 \mathrm{O} 8$ ", Physical Review Letters, (2003), Submitted.

4. Nodvick, J.S., Saxon, D.S., "Suppression of Coherent Radiation by Electrons in a Synchrotron", Physical Review, 96 (1954), 180.

5. Hirschmugl, C.J., Sagurton, M., Williams, G.P., "Multiparticle coherence calculations for synchrotron-radiation emission", Physical Review A, 44 (1991), 1316.

6. Andersson, A., Johnson, M. S., Nelander, B., "Coherent synchrotron radiation in the far infrared from a 1-mm electron bunch", Optical Engineering, 39 (2000), 3099.

7. Arp, U., G.T. Fraser, A.R.H. Walker, T.B. Lucatorto, K.K. Lehmann, K. Harkay, N. Sereno, and K.J. Kim, "Spontaneous coherent microwave emission and the sawtooth instability in a compact storage ring", Physical Review Special TopicsAccelerators \& Beams, 4 (2001), 054401.

8. Carr, G.L., Kramer, S. L., Murphy, J. B., Lobo, R.P.S.M., Tanner, D. B., "Observation of coherent synchrotron radiation from the NSLS VUV ring", Nuclear Instruments \& Methods in Physics Research Section A-Accelerators Spectrometers Detectors \& Associated Equipment, 463 (2001), 387.

9. Byrd, J.M., W.P. Leemans, A. Loftsdottir, B. Marcelis, M.C. Martin, W.R. McKinney, F. Sannibale, T. Scarvie, and C. Steier, "Observation of broadband 
self-amplified spontaneous coherent terahertz synchrotron radiation in a storage ring", Physical Review Letters, 89 (2002), 224801.

10. Stupakov, G. and S. Heifets, "Beam instability and microbunching due to coherent synchrotron radiation - art. no. 054402", Physical Review Special Topics-Accelerators \& Beams, 505 (2002), 4402.

11. Abo-Bakr, M., Feikes, J., Holldack, K., Wüstefeld, G., Hübers, H. W., "Steadystate far-infrared coherent synchrotron radiation detected at BESSY II", Physical Review Letters, 88 (2002), 254801.

12. Abo-Bakr, M., Feikes, J., Holldack, K., Kuske, P., Peatman, W.B., Schade, U., Wüstefeld, G., Hübers, H. W., "Brilliant, Coherent Far Infrared (THz) Synchrotron Radiation", Physical Review Letters, 90 (2003), 094801.

13. Sannibale, F., J.M. Byrd, A. Loftsdottir, M.C. Martin, and M. Venturini. "A Model for Producing Stable, Broadband Terahertz Coherent Synchrotron Radiation in Storage Rings", in Particle Accelerator Conference. 2003. Portland, OR.

14. Kuske, P. and G. Wustefeld, "Private Communication".

15. Li, D. and S. De Santis, "Private Communication". 
Table 1. List of performance parameters for the CSR emission of the designed CIRCE ring. Powers and energies are integrated from $1-100 \mathrm{~cm}^{-1}$ for the full 300 by $140 \mathrm{mrad}$ photon beam ports. When two values are presented as a range, they correspond to the red and green curves in Figure 4.

\begin{tabular}{cc} 
Parameter & Value \\
\hline Average Power & $13-311$ Watts \\
Peak Power & $3.5-28$ Kilowatts \\
Pulse Length & $\begin{array}{c}\geq 300 \text { femtoseconds } \\
\text { (transform limited) }\end{array}$ \\
Repetition Rate & Up to $1.5 \mathrm{GHz}$ \\
Energy per pulse & $9-210$ nanoJoules \\
Electron beam energy & $600 \mathrm{MeV}$ \\
Total beam current & $20-225 \mathrm{~mA}$ \\
Momentum compaction & $9.6 \times 10^{-5}-7.6 \times 10^{-4}$
\end{tabular}




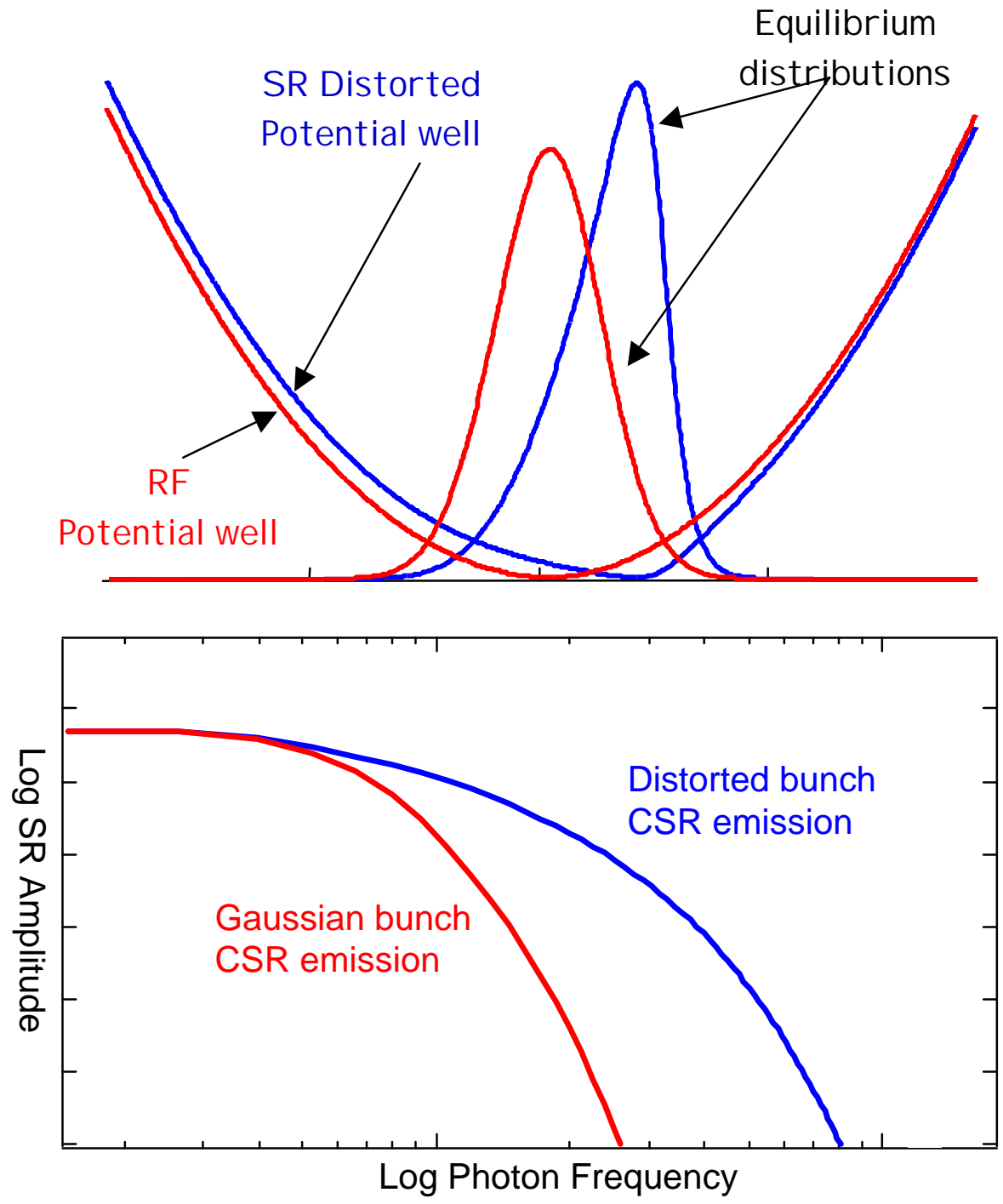

Figure 1. An electron bunch becomes non-Gaussian when the SR wakefield distorts the RF cavity potential well creating a bunch distribution that has a sharper leading edge (upper panel). This distorted bunch shape leads to CSR emission to higher frequencies than allowed by a Gaussian bunch (lower panel). 


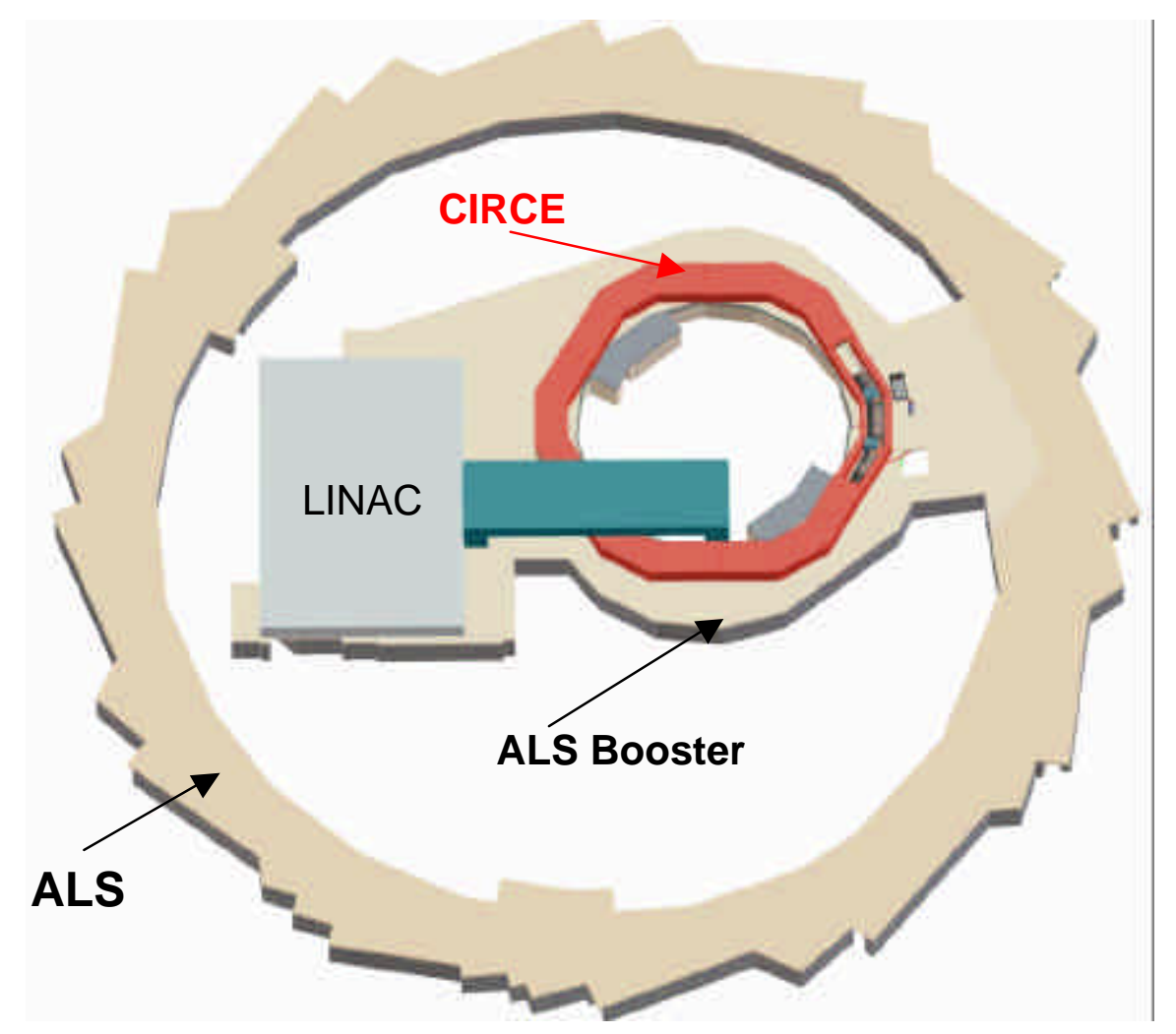

Figure 2. Location of the CIRCE ring on top of the existing ALS booster sheilding. The ring design allows for numerous CSR beamlines located directly next to the sheild walls (red color) and the parasitic use of the ALS injector for full-energy beam injection into CIRCE. 


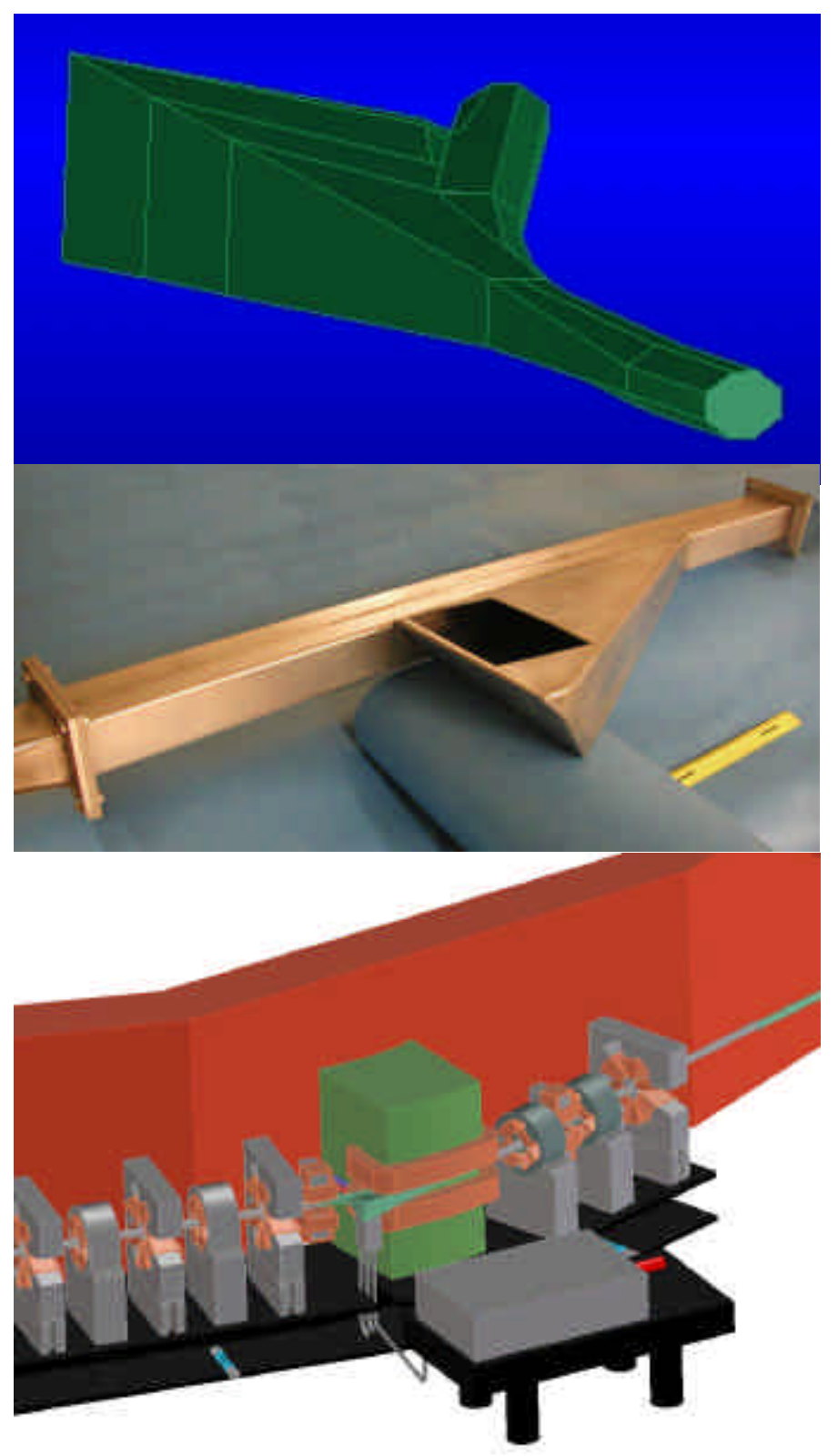

Figure 3. 3D CAD drawings of the photon beam extraction chamber (top) and of the chamber inserted into the dipole bending magnet with schematics of the beam transportation to a spectrometer beamline (bottom). A photograph of a mock chamber fabricated for RF testing is shown in the middle. The chamber is designed to collect $300 \mathrm{mrad}$ horizontally and $140 \mathrm{mrad}$ vertically. This very large acceptance means that the first mirror collects $95 \%$ of the radiation at $1 \mathrm{~mm}$ wavelength, so the CSR extraction is very efficient. 


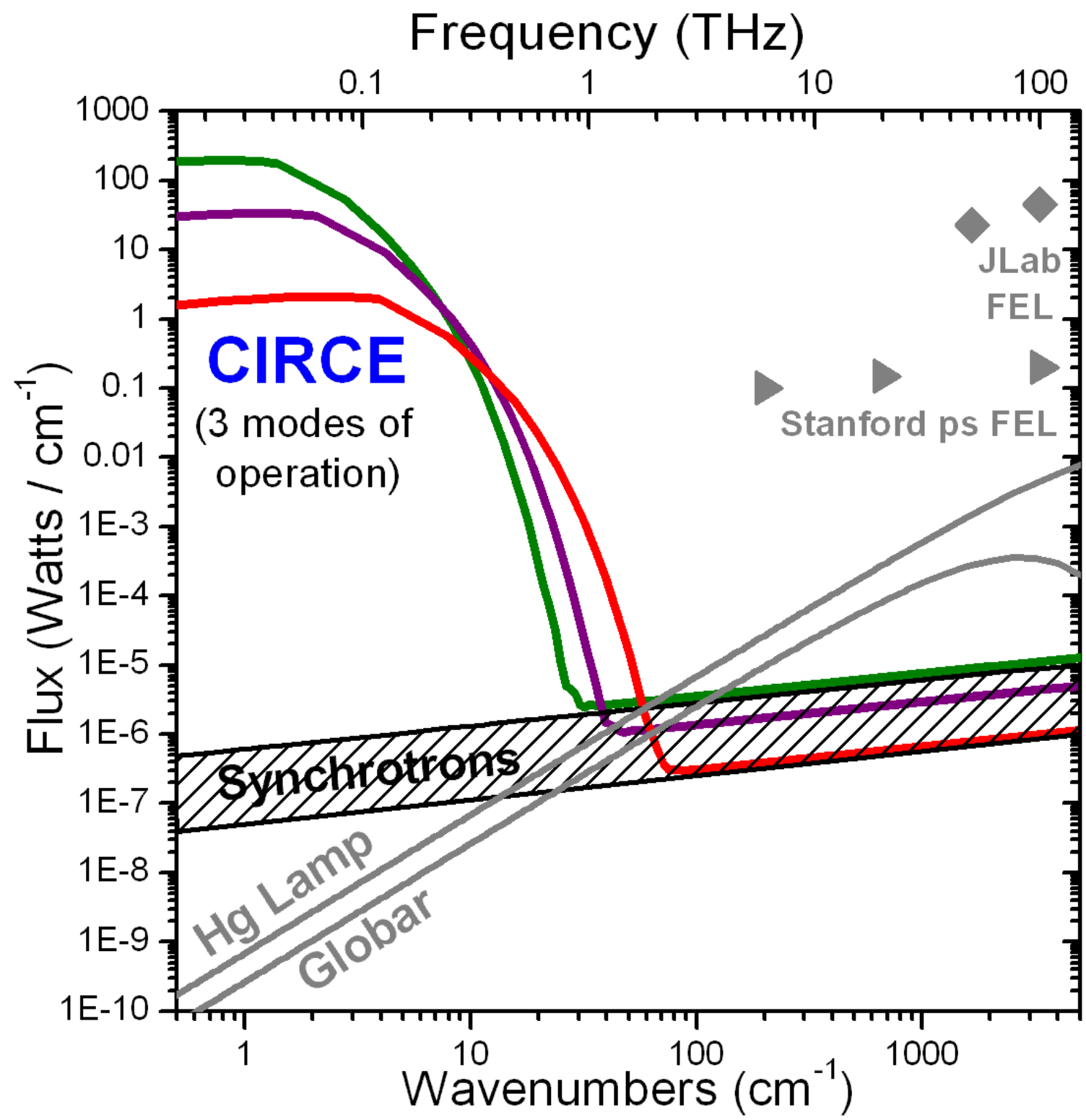

Figure 4. Flux calculations for CIRCE compared with conventional synchrotron and thermal far-IR sources, as well as two existing free electron lasers. The three CIRCE curves demonstrate how the CIRCE source can be tuned for high power (green curve), for extending coherent emission to shorter wavelengths (red curve), or somewhere in between (such as the purple curve). 